M2 occlusion stroke is unclear, and we studied the incidence of deterioration as well as the incidence of interventional treatment at our center.

Methods We retrospectively reviewed patients admitted to our comprehensive stroke center with an acute ischemic stroke due to occlusion of an M2 branch of the middle cerebral artery who underwent MRI brain during their hospitalization, and analyzed their clinical and imaging features. The M2 segment was defined as distal to the first bifurcating branch. FLAIR hyperintense vessels were defined as a linear hyperintensity present on two or more slices conforming to the location of the middle cerebral artery. Neurologic deterioration was defined as a decline by more than 4 NIHSS points or a sustained new neurologic deficit.

Results A total of 98 patients were treated at our center for M2 occlusion from 2012-2017. 71 patients (72\%) received an MRI brain during their hospitalization and were included in this analysis. FLAIR hyperintense vessels were present in a total of $30(42.3 \%) .20$ patients $(28.2 \%)$ deteriorated during their stay, and FHV were present in $12 / 20$ of these patients. Of the 51 patients who did not deteriorate, FHV were present in only 17 of them. Patients who deteriorated appeared to be more likely to have FHV than those who did not deteriorate $(47 \%$ difference, CI 21.4243-63.4427, $\mathrm{p}=$ 0.0004). Figure 1 demonstrates the presence of FHV in a patient which resolved after mechanical thrombectomy, supporting the hypothesis that FHV represents the ischemic penumbra.

Conclusion FHV may be a risk factor for neurologic decline in patients with M2 occlusion, though more data is needed to determine the utility of FHV in assessing risk of neurologic worsening. Our analysis did not take into account differences between treated and untreated patients; prior studies have suggested FHV correlates with ischemic penumbra and may correlate with better collateral circulation. Further studies are needed to correlate FHV with perfusion imaging and outcome adjusted for treatment.

Disclosures K. Dakay: None. M. Jayaraman: None. R. McTaggart: None. S. Yaghi: None. G. Jindal: None. S. Cutting: None.

\section{E-056 CURRENT APPLICATIONS OF OCT IN NEUROINTERVENTIONAL ANGIOGRAPHY: A RETROSPECTIVE CROSS-SECTIONAL OBSERVATIONAL ANALYSIS}

E Luther ${ }^{*}$, R Starke. University of Miami Department of Neurological Surgery, Miami, FL

10.1136/neurintsurg-2019-SNIS.131

Introduction Optical coherence tomography (OCT) is a novel intraluminal imaging modality that uses optical backscattering to produce cross-sectional images of tissue. The technique has already proven useful in the coronary vasculature for evaluation of plaques, stent-vessel interactions, dissections and intimal healing. However, there is limited data regarding the current applications of OCT in cerebrovascular imaging.

Methods We performed a retrospective analysis of the Nationwide Inpatient Sample (NIS) between 2009-2015. Patients with an ICD-9-CM procedure code indicating use of OCT (38.25) and an ICD-9-CM diagnosis code indicating either presence of an intracranial aneurysm/SAH (437.3 or 430), carotid/vertebrobasilar/intracranial atherosclerosis with or without associated cerebral infarction (433.xx or 434.xx), or carotid/vertebrobasilar arterial dissection (443.21 or 443.24) were included. Primary diagnosis codes, primary procedure codes, hospital region, hospital teaching status, rate of iatrogenic neurologic injury, and time to use of OCT were evaluated in an effort to identify current inpatient OCT practices in neurointerventional angiography.

Results 7 patients in the NIS were identified as having undergone OCT for a cerebrovascular pathology between the years of 2009-2015. Average age was 71.3 years. 2 patients were female and only $28 \%$ were elective admissions. 4 patients were discharged home, 2 would be discharged to a long-term care facility and 1 patient would require home health care. The two most common primary diagnoses were carotid artery occlusion/stenosis with or without mention of cerebral infarction (433.11 and 433.10, respectively). The two most common primary procedures were diagnostic cerebral angiography and carotid endarterectomy (88.41 and 38.12, respectively). Median LOS was 3 days. The only hospital regions represented were the Northeast and the South and those in the South were solely performed at urban non-teaching hospitals while those in the Northeast were only at urban teaching hospitals. Interestingly, OCT was performed on the same day as all primary procedures except for one patient who underwent OCT two days prior to undergoing elective carotid endarterectomy. Interestingly, this was also the only patient who experienced iatrogenic cerebral infarction/hemorrhage during the admission.

Conclusions Although OCT has proven to be an effective adjunct imaging modality in the coronary vasculature, few neurointerventionalists have adopted it since it received FDAapproval in 2010. Moreover, it appears that those who have begun to use it have only applied it to atherosclerotic disease despite the fact that several animal and cadaveric studies have proven it effective in identifying both aneurysm neck morphology and endothelialization post-embolization. The utility and efficacy of OCT in various cerebrovascular pathologies warrant further investigations.

Disclosures E. Luther: None. R. Starke: None.

\section{E-057 ADMISSION CT PERFUSION MAY UNDERESTIMATE INITIAL INFARCT CORE IN ISOLATED DEEP MIDDLE CEREBRAL ARTERY STROKES}

M Bouslama*, K Ravindran, G Rodrigues, L Pisani, D Haussen, C Barreira, M Frankel, R Nogueira. Emory University/Grady Memorial Hospital, Atlanta, GA

10.1136/neurintsurg-2019-SNIS.132

Background and purpose Proper identification of infarct core is crucial in establishing stroke prognosis and determining whether a patient might be a candidate for endovascular therapy (ET). We sought to study those cases in which admission CTP misses a core lesion that is present on initial non-contrast CT.

Methods Review of a prospectively collected database of endovascular patients with anterior circulation Large vessel occlusion strokes from January 2014-November 2018. Only patients with an e-ASPECT score $<10$ and adequate CTP maps were included. A Total Missed infarct Core (TMC) was defined as a CTP core lesion $(\mathrm{rCBF}<30 \%)<1 \mathrm{cc}$ with a visualized hypodensity on initial non-contrast CT. 